\title{
Conditionally replicating adenovirus SG500-expressed mutant Dm-dNK gene for breast cancer therapy
}

\author{
WENZHI QU ${ }^{1,2}, \mathrm{ZHI} \mathrm{ZHU}^{2}$, $\mathrm{LEI} \mathrm{ZHAO}^{4}, \mathrm{ANNING} \mathrm{HE}^{3}$ and XINYU ZHENG ${ }^{2,3}$ \\ ${ }^{1}$ Department of Breast Surgery, Fourth Affiliated Hospital; ${ }^{2}$ Department of Surgical Oncology, \\ Department of General Surgery, First Affiliated Hospital; ${ }^{3}$ Laboratory 1, Cancer Institute; \\ ${ }^{4}$ Center of Experiment Technology and Medical Research, China Medical University, Shenyang, P.R. China
}

Received July 10, 2012; Accepted September 3, 2012

DOI: 10.3892/ijo.2012.1657

\begin{abstract}
The purpose of this analysis was to investigate the enzyme activity and specificity of using adenovirusmediated Drosophila melanogaster deoxyribonucleoside kinase (Dm-dNK) mutants in combination with gemcitabine. Compared with herpes simplex type 1 thymidine kinase (HSV-TK) and other known dNKs, this Dm-dNK enzyme has a broader substrate specificity and a higher catalytic rate. We created the Dm-dNK mutants (dNKmu) by site-directed mutagenesis at the sites of $244 \mathrm{E}, 245 \mathrm{~S}, 251 \mathrm{~S}$ and $252 \mathrm{R}$, with the last 10 amino acids in the amino acid sequence randomly mutated. We evaluated the enzyme activity and substrate specificity. The engineered enzymes showed a relative increase in phosphorylation in the nucleoside analogs of BVDU ((E)-5-(2-Bromovinyl)-2'-deoxyuridine) or gemcitabine (DFDC, 2',2'-difluoro-deoxycytidine) compared with the wild-type enzyme. The dNKmu enzymes were expressed in the breast cancer cell lines MDA-MB-231 (ER-) and MCF7 $\left(\mathrm{ER}^{+}\right)$. In studying the sensitivity of the cell lines to DFDC, conditionally replicative adenovirus (CRAd) SG500-dNKmu showed higher expression and enzymatic activity than the replication-defective adenovirus SG500 in cancer cells, but with less cytotoxicity to cancer cells than that of SG500. Our data suggest that the triple phosphorylated DFDC catalyzed by dNKmu inhibited the replication of adenovirus with a simultaneous positive therapeutic effect to cancer cells. Therefore, concomitant use of the SG500-dNKmu and DFDC could be a novel targeted strategy in suicide gene therapy with safe control of excessive virus replication.
\end{abstract}

Correspondence to: Dr Xinyu Zheng, Department of Surgical Oncology, Department of General Surgery, First Affiliated Hospital, China Medical University, 155 North Nanjing Street, Shenyang 11001, P.R. China

E-mail: xyzheng@mail.cmu.edu.cn

Key words: Drosophila melanogaster multisubstrate deoxyribonucleoside kinase, oncolytic adenovirus, virus therapy, suicide gene therapy, safe control

\section{Introduction}

Breast cancer is one of the common malignant tumors of females. According to recent statistics, breast cancer occupies the first place among newly diagnosed tumor of American women. In China, the incidence of breast cancer has increased steadily and the patients tend to be younger in the past decade. Surgical resection in combination with systemic chemotherapy in breast cancer patients is effective, but the effect is not satisfactory. Gene therapies of cancer are unique in offering the possibility of treatments that eradicate tumors and metastases without damaging normal tissues. The system combined with chemotherapy of breast cancer in vitro and in vivo show that this vector system treatment with targeting TRE (tetracycline-responsive element) promoter combined with appropriate chemotherapy are more effective than either of them alone.

The multisubstrate deoxyribonucleoside kinase of Drosophila melanogaster (Dm-dNK) has been used as a novel suicide gene and evaluated for its effectiveness in a number of cancer cell lines. Based on previous findings, expression of Dm-dNK in cancer cells increases the sensitivity to several cytotoxic nucleoside analogs (1-4), rendering the enzyme a candidate for possible use as a suicide gene with combined gene therapy and chemotherapy.

Aligned with other deoxyribonucleoside kinases, homologous amino acid patches are distributed over the entire amino acid sequence of Dm-dNK, except for the C-terminal part. The last 10 amino acids of the very C-terminal part are unique and not only influence catalytic efficiency for thymidine but also involve nuclear localization signals. The role of the $\mathrm{C}$-terminal part of the enzyme was investigated in detail by construction and expression of a 10 amino-acid deletion mutant. The $\Delta 10$ Dm-dNK mutant has an even higher catalytic rate for deoxyribonucleosides compared with the wild-type enzyme (5). To further explore the phosphorylation capacity of mutagenesis, we designed and constructed mutated Dm-dNK (Dm-dNKmu) with the last 10 amino acids randomly alternated at the sites of 244E, 245S, 251S and 252R in the amino-acid sequence. We wished to study its enzymatic properties in a functional assay after cellular expression.

Human telomerase reverse transcriptase (hTERT) is expressed in cells of telomerase activity during immortaliza- 
tion process, telomerase is activated in most human malignant cells but cannot be detected in normal somatic cells. Activation of hypoxia response element (HRE) promoter causes specific changes of solid tumors, it leads to exogenous gene expression in tumor cells, but minimal expression in normal cells (6-8). Accordingly it can be used as the tumor-specific targeting promoter. hTERT and HRE are added, respectively, to early transcription units E1A and E1B of conditionally replicating adenovirus SG500 (9). Like ONYX-015, SG500 can replicate in p53-disfunctional tumor cells and kill them. Containing cloning sites, SG500 can insert exogenous gene to form SG500-gene, while ONYX-015 cannot. So as a carrier, the SG500 enables exogenous gene targeting transcription in tumor cells achieving multiple targeting.

BVDU and DFDC is known to enhance cell killing activity by phosphorylation of enzymes $(10,11)$. However, our use of mutants combined with them is yet to be studied in suicide gene therapy, or in an in vivo animal model. Therefore, we constructed the $\mathrm{dNKmu/BVDU}$ or DFDC system to evaluate the efficacy of our approach in the prevention of breast cancer. In this study, we took advantage of the conditionally replicative adenovirus (CRAd) SG500 vector. Conditionally replicating adenovirus SG500 introduced Dm-dNK mutants (dNKmu) into breast cancer cell lines MDA-MB-231 and MCF-7, recording tumor cell killing efficiency after adding different concentrations of nucleoside and testing killing effect and safety of SG500-dNKmu at different levels. Then we performed the tumor-bearing mice tests in vivo to observe the safety and sensitivity of SG500-dNKmu nucleotide analogs in animal experiments, and investigated the breast cancer cell killing effect of this treatment system to provide new ideas for breast cancer treatment.

\section{Materials and methods}

Cells and cell cultures. The human breast cancer cell line, MDA-MB-231 and MCF-7, were both purchased from the Institute of Cell Biology, Chinese Academy of Sciences (Shanghai, China). MRC-5 and WI-38, the human embryo lung fibroblast cell line and HEK293, the human embryonic kidney cell line, were both purchased from American Type Culture Collection (Manassas, VA, USA). MDA-MB-231 and MCF-7 were cultured in Dulbecco's modified Eagle's medium (DMEM, Gibco, Grand Island, NY, USA) supplemented with $10 \%$ heat-inactivated fetal bovine serum (FBS, Gibco) and incubated at $37^{\circ} \mathrm{C}, 5 \% \mathrm{CO}_{2}$. WI-38, MRC-5 and HEK293 were cultured in MEM (Gibco) or RPMI-1640 (Gibco) supplemented with $10 \%$ heat-inactivated FBS and incubated at $37^{\circ} \mathrm{C}, 5 \% \mathrm{CO}_{2}$.

Construction of recombinant adenovirus. The complete cDNA sequence of Dm-dNKmu gene was released from plasmid pGEM-T-dNKmu with endonucleases EcoRI and BamHI (New England Biolabs, Beverley, MA, USA) and ligated into plasmid pENTR13 [made by ourselves, which contains the sequence of mouse cytomegalovirus (CMV) promoter + His-tag $(\mathrm{N})+$ multiple clone site $+\mathrm{SV} 40$ polyA] to generate pENTR13-dNKmu. pENTR13-dNKmu was digested with endonucleases AgeI and NotI (New England Biolabs), a 1317 bp fragment expression cassette of $\mathrm{dNKmu}$ containing mouse CMV promoter $+\mathrm{dNKmu}$ gene $+\mathrm{SV} 40$ poly A was excised and inserted into the AgeI and NotI sites of pSG500 (Sinogenomax Inc., Beijing, China) to generate pSG500-dNKmu. Plasmid pSG500-dNKmu was individually transfected into HEK293 cells using Lipofectamine 2000 (Life Technologies) combined with adenovirus packaging plasmid pBGHlox (Microbix Biosystems). After homologous recombination, we obtained a replicative adenovirus known as SG500-dNKmu, in which the viral E1B55-kDa gene was deleted. Replication-defective adenovirus Ad-GFP is used to compare infection rate differences between the cell lines.

RT-PCR analysis. MDA-MB-231, MCF-7, WI-38 and MRC-5 cells were infected with SG500-dNKmu or SG500 at a multiplicity of infection (MOI) rate of 10 . Two days after infection, cells were purified with a TRIzol (Sigma, St. Louis, MO, USA) method and then assayed for Dm-dNKmu gene expression using the RT-polymerase chain reaction (PCR) kit (Takara Bio Inc., Japan). This manipulation was done according to the manufacturer's instructions. A cDNA equivalent of $1 \mathrm{ng}$ of RNA was amplified by PCR using primers specific for the target genes. The thermal cycles were: $94^{\circ} \mathrm{C}$ for $1 \mathrm{~min}, 55^{\circ} \mathrm{C}$ for $1 \mathrm{~min}, 72^{\circ} \mathrm{C}$ for $1.5 \mathrm{~min}$ for 35 cycles for dNKmu (785 bp); and $94^{\circ} \mathrm{C}$ for $1 \mathrm{~min}, 55^{\circ} \mathrm{C}$ for $1 \mathrm{~min}, 72^{\circ} \mathrm{C}$ for $1.5 \mathrm{~min}$ for 35 cycles for GAPDH (452 bp). Nucleotide sequences of dNKmu primers were as follows: sense 5'-AAGGACTGATGGCGGAGGCA-3'; antisense 5'-TTGTCGTACCTGGCGACCCTCTGGCT-3'. Nucleotide sequences of GAPDH primers were as follows: sense 5'-ACCA CAGTCCATGCCATCAC-3'; antisense 5'-TCCACCACCCTG TTGCTGTA-3'. The amplification products were separated by $2 \%$ agarose gel electrophoresis and visualized by SYBR-Green staining.

Immunoblot analysis. MDA-MB-231, MCF-7, WI-38 and MRC-5 cells were seeded in 24-well plates at a density of $5 \times 10^{4}$ cells/well and infected with SG500 or SG500-dNKmu at a MOI of 1 . After 2 days, cells were rinsed 3 times with phosphate-buffered saline (PBS) and fixed in $4 \%$ paraformaldehyde for $25 \mathrm{~min}$. Immunoblotting was performed by incubation, first with mouse anti-human His-tag antibody (Merck, Darmstadt, Germany) at $4^{\circ} \mathrm{C}$ for $1 \mathrm{~h}$, followed by incubation with goat anti-mouse IgG (Santa Cruz Biotechnology, Santa Cruz, CA, USA) at a dilution of 1:100.

Enzyme assay. MDA-MB-231, MCF-7, WI-38 and MRC-5 cells were seeded in 6 -well plates at a density of $5 \times 10^{5}$ cells/well and cultured for $24 \mathrm{~h}$, followed by infection with SG500-dNK and SG500 at a MOI of 1. Two days later, cell protein extracts were prepared as described (12). The assays were performed in $50 \mathrm{mM}$ of Tris- $\mathrm{HCl}, \mathrm{pH} 7.6,5 \mathrm{mM}$ of $\mathrm{MgCl}_{2}, 5 \mathrm{mM}$ of ATP, $2 \mathrm{mM}$ of dithiothreitol, $15 \mathrm{mM}$ of $\mathrm{NaF}, 100 \mathrm{mM}$ of $\mathrm{KCl}, 0.5 \mathrm{mg} / \mathrm{ml}$ bovine serum albumin, and $0.6 \mathrm{mg}$ of protein extract in a total volume of $35 \mathrm{ml}$. We used $2.5 \mathrm{mM}$ of methyl-3H-dThd (Moravek Biochemicals, Brea, CA, USA) in the assays and mixed with equivalent amounts of unlabeled substrates. Aliquots of the reaction mixture were spotted on Whatman DE-81 filters after 10, 20 and $30 \mathrm{~min}$ incubation at $37^{\circ} \mathrm{C}$. The filters were washed 3 times in $5 \mathrm{mM}$ 
of ammonium formate. The nucleoside monophosphates were eluted from the filter with $0.5 \mathrm{M}$ of $\mathrm{KCl}$ and the radioactivity was determined by scintillation counting.

Cell killing assay. Cells were dispensed in 96-well plates (Corning Inc., Corning, NY, USA). The culture solution was removed on the second day and the following were added to each well: $1 \mathrm{ml}$ of serum-free DMEM and virus SG500-dNKmu, SG500 and mock at a MOI of 10. After 2 days of incubation, the nucleoside pro-drug BVDU or DFDC was added at a concentration of $0,0.001,0.01,0.1,1$ and $10 \mu \mathrm{M}$ (mock was the drug-treated only group). After another 3 days, and a total of 5 days of incubation, cell viability was measured by the tetrazolium salt 3-(4,5-dimethylthiazol-2-yl) -2,5-diphenyl tetrazolium bromide (MTT) assay (Promega, Madison, WI, USA) to determine the combined cytotoxic effect. Absorbance was measured with an Enzyme Immunoassay Instrument at $570 \mathrm{~nm}$, which serves as a measure of cell viability. Each data point was generated from triplicate samples and repeated 3 times.

Flow cytometry apoptosis assays. For further clarification of the mechanism and effect of the combined suicide therapy, the apoptotic cells were detected by an Annexin V-FITC/PI double staining kit (Genmed Bioscience, China). Briefly, MDA-MB-231 and MRC-5 cells were treated with $1 \mu \mathrm{M}$ of DFDC for 3 days after being infected with SG500-dNKmu, SG500 and Mock for $48 \mathrm{~h}$, then trypsinized, pelleted and washed in PBS. Cells were rinsed with a binding buffer and then resuspended with $200 \mu \mathrm{l}$ of the binding buffer. After adding $5 \mu \mathrm{l}$ of Annexin V-FITC (20 $\mu \mathrm{g} / \mathrm{ml})$ and $10 \mu \mathrm{l}$ of propidium iodide (PI, $50 \mu \mathrm{g} / \mathrm{ml}$ ), the mixture was incubated at room temperature for $10 \mathrm{~min}$ in the dark. The apoptosis ratio was analyzed using a FACScan flow cytometer [equipped with Cellquest and ModFITLT for Mac V1.01 software (Becton-Dickinson, San Jose, CA, USA)].

Adenovirus replication assay. MDA-MB-231, MCF-7 and MRC-5 were cultured in 24-well dishes overnight and infected with SG500-dNKmu, SG500 and wild-type Ad at an MOI of 10, then divided into two groups. One group was treated with $1 \mu \mathrm{M}$ of DFDC for 3 days. Two days after infection, a second group was only virus-treated for 5 days. Cells and media were then collected, respectively, freeze-thawed and titered on HEK293 cells by the limiting dilution method (TCID50). Each assay was repeated 2 to 3 times, averaged, and expressed as pfu/cell \pm SD.

Western blot analysis. Subconfluent cells were infected with viruses in the presence of BVDU or DFDC as described above. MDA-MB-231 and MRC-5 cells were harvested 5 days postinfection and lysed in buffer $(50 \mathrm{mmol} / 1$ herpes, at $\mathrm{pH} 7.4$, $250 \mathrm{mmol} / 1 \mathrm{NaCl}, 1 \mathrm{mmol} / 1 \mathrm{EDTA}, 1 \mathrm{mmol} / \mathrm{l} \mathrm{DTT}, 1 \mathrm{mmol} / 1$ $\mathrm{NaF}, 1 \%$ Triton $\mathrm{X}-100)$ containing protease inhibitors. Total protein (50-80 $\mu \mathrm{g}$ ) was separated on SDS-polyacrylamide gels under reducing conditions, transferred to polyvinylidene fluoride membranes (Millipore), and detected by the following antisera: rabbit anti-Ad2/Ad5 E1A at 1:1,000 dilution (Santa Cruz Biotechnology) and goat anti- $\beta$-actin at 1:1,000 dilution (Santa Cruz Biotechnology), mouse anti-human His-tag at 1:500 dilution (Merck). Detection was by horseradish peroxidase-conjugated secondary IgG-antibodies (Santa Cruz Biotechnology) as appropriate, and chemiluminescence reagent (Amersham Pharmacia Biotech) followed by autoradiography (BioMaxfilm, Kodak).

In vivo tumor growth. Female BALB/C nude mice 4- to 6-week-old were bred and maintained under specific pathogenfree conditions, and all studies conducted under the protocols approved by the China Medical University of Shenyang Animal Research Committee. Mice were subcutaneously injected with breast cancer cells (MDA-MB-231, $10^{7}$ cells suspended in $100 \mu \mathrm{l}$ PBS) into the right flank of each of the 48 mice. When the tumors had grown to about $90 \mathrm{~mm}^{3}$ in size, the mice were randomly divided into 6 treatment groups: Group 1, DFDC with SG500-dNKmu and Group 2, SG500 infected; Group 3, SG500-dNKmu and Group 4, SG500 virus alone; Group 5, DFDC alone and PBS (control) group. All of the mice in the adenovirus treatment groups were intratumoraly injected with a total dose of $10^{9} \mathrm{pfu} 3$ times at 48 -h intervals and DFDC at $5 \mathrm{mg} / \mathrm{kg}$ i.p. twice on days 2 to 8 after virus injection. Tumor growth was monitored 5 days once and tumor volume $(\mathrm{V})$ was calculated using the formula: $\mathrm{V}\left(\mathrm{mm}^{3}\right)=1 / 2$ length $(\mathrm{mm}) \mathrm{x}$ width $(\mathrm{mm})^{2}$. Survival analysis expressed as time to progression was performed according to the method of Kaplan-Meier (log-rank test for statistical significance). Tumor growth curves were compared using one-way ANOVA for significance.

\section{Results}

Construction of recombinant adenovirus with Dm-dNKmu. Mutations of Dm-dNK at the last 10 amino acids in the sequence may increase the sensitivity of cells to other purine nucleoside analogs as well as altering the expression location of Dm-dNK. The Dm-dNK mutants were created by site-directed mutagenesis in the amino-acid sequence at sites of 244E, 245S, 251S and $252 \mathrm{R}$ alternated to evaluate the enzyme activity (Fig. 1A). The viral E1B55-kDa gene was replaced by an expression cassette encoding dNKmu containing the sequence of $\mathrm{CMV}$ promoter + multiple clone site + SV40 poly A into plasmid SG500 to generate plasmid SG500-dNKmu (Fig. 1B). The infectivity of the cell lines were determined by the wild-type 5 adenovirus with expression of green fluorescence (Ad5-GFP), as shown in Fig. 1C. The cancer cell lines MDA-MB-231 and MCF-7 and the normal cell line WI38 and MRC-5 exhibited nearly the same infectivity.

Expression and enzyme activity of Dm-dNKmu.SG500-dNKmu expressed the Dm-dNKmu gene with a higher efficiency and activity in MDA-MB-231 and MCF-7 cancer cells compared with the replication-deficient adenovirus SG500. Our RT-PCR study results showed the selective expression of SG500-dNKmu at a MOI of 10 after a 2-day infection. The dNKmu gene of SG500-dNKmu was somewhat higher than that of SG500 in MDA-MB-231 and MCF-7 cancer cells; however, in MRC-5 cells, SG500-dNKmu showed lower levels than that of SG500. (Fig. 2A). Enzyme activity was determined by the phosphorylation of dThd. The uninfected or infected with SG500 vector adenovirus showed low levels of dThd phosphorylation, whereas the MDA-MB-231 or MCF-7 cancer cells infected with SG500dNKmu exhibited nearly 30 -fold higher enzymatic activity than 
A

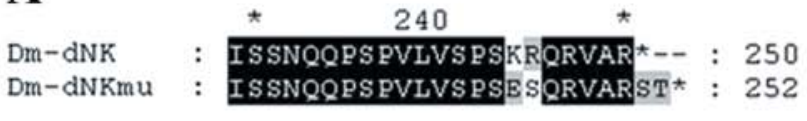

C

\section{MDA-MB-231}

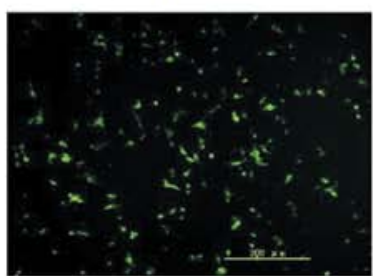

MRC-5

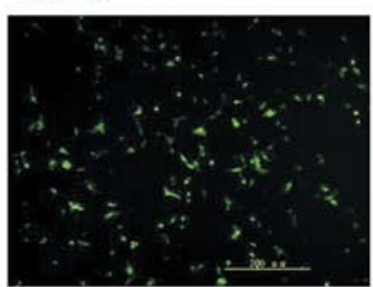

B

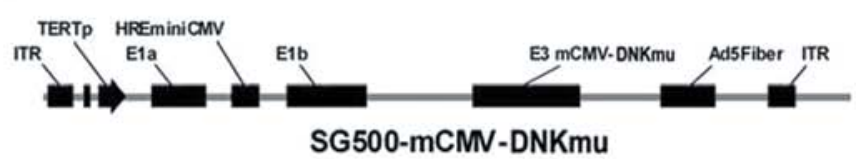

MCF-7
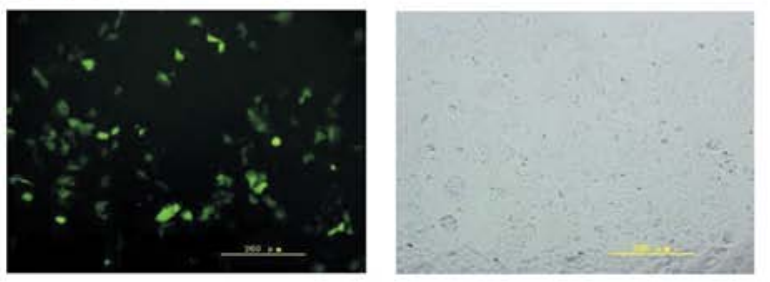

WI-38
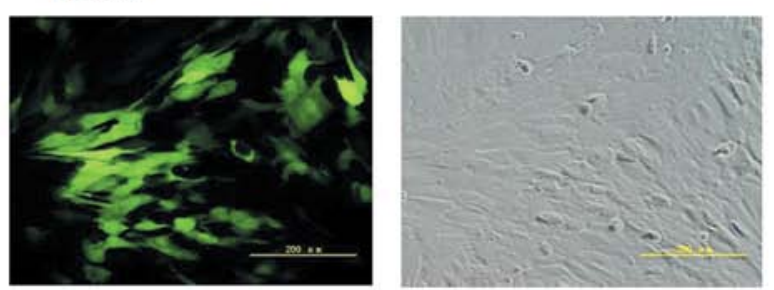

Figure 1. Construction and infectivity of SG500-dNKmu and SG500. (A) Shows multiple alignment of the predicted amino acid sequences of Dm-dNK and Dm-dNKmu, but the last 10 amino acids were randomly alternated at site of 244E, 245S, 251S and 252R. Amino acid sequences were obtained from the NCBI database. (B) To construct and infect SG500-dNKmu, the E1B55-kDa gene was replaced by the expression cassette of Dm-dNKmu containing CMV promoter + multiple clone site + SV40 poly A. The expression cassette was also inserted to the pShuttle vector to generate SG500. ITR, inverted terminal repeat; $\psi$, the adenovirus 5 packing signal; PUC origin, prokarotic origin of replication. (C) An overview of the infectivity in the 4 cell lines (MOI=100). More than 90\% of the cells expressed GFP at MOI=100 with Ad5-GFP infected $48 \mathrm{~h}$ later.

A

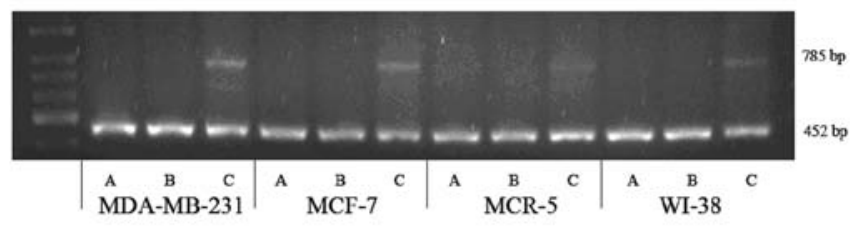

B

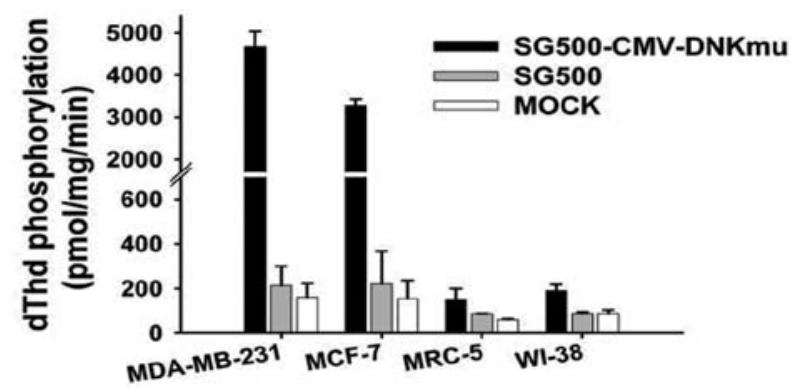

Figure 2. Expression and enzyme activity of Dm-dNKmu. Dm-dNKmu expression was quantified by (A) RT-polymerase chain reacton (PCR) analysis Dm-dNKmu expression in MDA-MB-231, MCF-7 and MRC-5 cells infected with (a) mock, (b) SG500 and (c) SG500-dNKmu at MOI=10 for $48 \mathrm{~h}$ after infection. The vector infected cells were used as control. 785 bp and $452 \mathrm{bp}$ indicate the size of the amplified Dm-dNKmu and GAPDH. (B) The nucleoside kinase activity was quantified by the level of phosphorylation of dThd. The cells uninfected or infected with SG500 adenovirus showed low levels of dThd phosphorylation, whereas the cancer cells infected with SG500-dNKmu exhibited nearly 30 -fold higher enzymatic activity than the parent cell line, 5 -fold higher than that of SG500 and 1.5-fold higher than that of SG500-dNK. The normal cell lines WI-38 and MRC-5 still showed stable low activity. the parent cell line; in the normal cell lines MRC-5 or WI-38, activity of cells infected by SG500-dNKmu was 10-fold less than that in cancer cells (Fig. 2B).

Cell cytotoxic activity of Dm-dNKmu with chemotherapy. SG500-dNKmu virus alone showed greater killing activity on cell lysis than DL1520 (ONYX-15) and WtAd in MDA-MB-231 cells, and almost the same protective as SG500 and DL1520 in MRC-5 cells (Fig. 3A). SG500-dNKmu induced much more rapid cell death in MDA-MB-231 cells than in normal MRC-5 cells 5 days after infection. Compared with DL1520 and WtAd infection, SG500-dNKmu demonstrated no more apparent cytolysis against MDA-MB-231 and MCF-7, but less profound cytotoxicity than DL1520 and WtAd5 against normal cells.

We investigated Dm-dNKmu for its potential use as a suicide gene in combination with the nucleoside analog BVDU or DFDC. As shown by the MTT assay results, the treatment of cells with SG500-dNKmu and SG500 resulted in a dose-dependent reduction of cell viability within 5 days. With different concentrations of BVDU or DFDC, SG500-dNKmu and SG500 were able to kill MDA-MB-231 and MCF-7 cancer cells more effectively than the SG500 and mock. This occurred even at the very low concentration of $0.001 \mu \mathrm{M}$ DFDC $(\mathrm{p}<0.05)$. The nucleoside analog dose used was much lower than $\mathrm{IC}_{50}$. Cell killing rates of SG500-dNKmu was slightly less than that of SG500 in MDA-MB-231 and not less than that of SG500 in the MCF-7 cell line. The difference may be because of the different enzyme activity and sensitivity in various cell lines. However, in the normal MRC-5 and WI-38 cells, SG500-dNKmu with drugs 

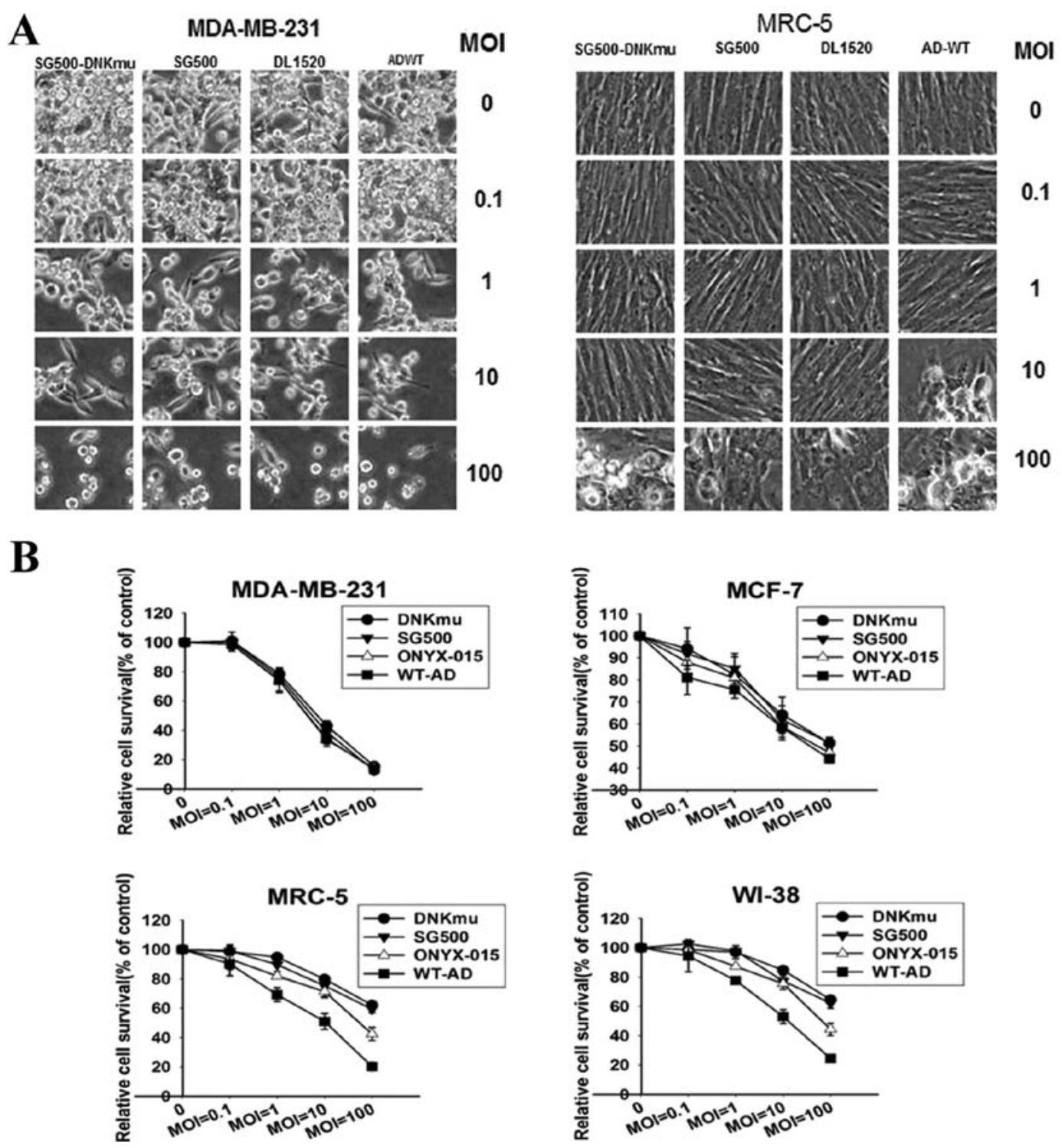

Figure 3. Selective replication of SG500-dNKmu. (A) The cytopathogenic effect was observed to determine whether SG500-dNKmu or SG500 induces selective cell lysis with MOI=1 infection. SG500-dNKmu showed greater killing activity on cell lysis than DL1520 (ONYX-15) and WtAd in MDA-MB-231 cells, and almost the same protective as SG500 and DL1520 in MRC-5 cells. (B) SG500-dNKmu induced much more rapid cell death in MDA-MB-231 cells than in normal MRC-5 cells 5 days after infection. Compared with DL1520 and WtAd infection, SG500-dNKmu demonstrated no more apparent cytolysis against MDA-MB-231 and MCF-7, but less profound cytotoxicity than DL1520 and WtAd5 against normal cells, furthermore, SG500-dNKmu and SG500 act almost the same on the cytotoxic effect to cancer cells or the safety to normal cells.

showed less cytotoxic than the other virus (Fig. 4A), with cell killing rates reaching a maximum of 50\% lower than SG500, even when drugs was added. The FACS assay also supported the results of MTT (Fig. 4B). The SG500-dNKmu and SG500 (MOI=10) with BVDU or DFDC induced the most cell apoptosis reaching as high as 61 and $82 \%$ (right upper and lower quadrant) in the MDA-MB-231 cancer cells, which was $60 \%$ higher than the SG500 with DFDC. On the other hand, in the MRC-5 cells, with the presence of $1 \mu \mathrm{M}$ of DFDC, $16 \%$ of apoptosis was induced by SG500-dNKmu, which was far less than SG500 and SG500. The above data suggest that SG500-dNKmu can sensitize cancer cell lines to DFDC without causing significant toxicity to normal cells.

Viral replication is inhibited by Dm-dNKmu with drugs. Surprisingly, in all the cell lines, viral replication was detected at very low levels in the presence of $1 \mu \mathrm{mol} / 1$ of DFDC for the combination with SG500-dNKmu, but not with SG500 without Dm-dNKmu. MDA-MB-231 cells supported higher levels of $10^{9} \mathrm{pfu} / \mathrm{ml}$ replication of the virus when SG500 was used alone, but in the presence of $1 \mu \mathrm{M}$ DFDC, replication was detected at low levels of $<10^{6} \mathrm{pfu} / \mathrm{ml}$ for the SG500-dNKmu (Fig. 5A). Despite a clear increase in replication over time without the drug, low doses of DFDC $(1 \mu \mathrm{M})$ added $48 \mathrm{~h}$ after infection can lead to replicative inhibition of the virus for the combination SG500dNKmu. Attenuation was also observed to varying degrees in normal cell lines. The normal cells did not support replication of SG500 vectors, but in combination with $1 \mu \mathrm{mol} / 1$ DFDC, viral replication was potently attenuated. The E1A protein levels in western blot experiments verify that DFDC activated by Dm-dNKmu led to inhibition of adenovirus replication. The E1A protein produced from the combination of SG500-dNKmu 

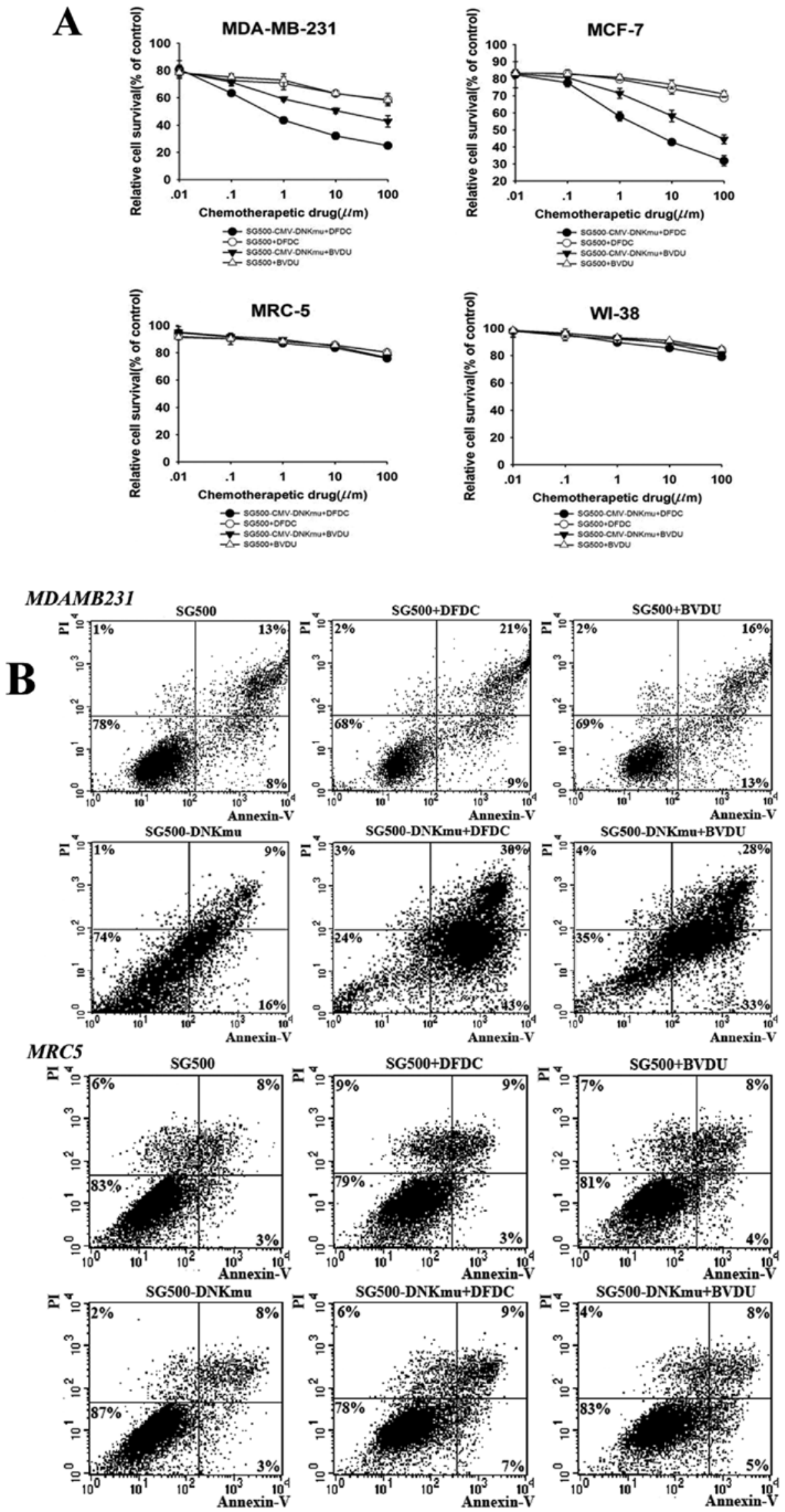

Figure 4. Selective cell killing activity of Dm-dNKmu with DFDC. (A) Cytotoxicity of Dm-dNKmu was assessed by MTT assay. Each cell line was infected with SG500-dNKmu or SG500 at a MOI of 10 in combination with DFDC at does ranging from 0.01-10 $\mu \mathrm{M}$. SG500-dNKmu induced nearly equal proportions of cell death in cancer cells to that of SG500, but less profound cytotoxicity against normal cells. (B) The MDA-MB-231 and MRC-5 cells were infected with SG500dNKmu, SG500, SG500 and Ad-blank (MOI 10) for 2 days, and DFDC $(1 \mu \mathrm{M})$ added for 3 days. After the treatment, the percentage of apoptotic cells death was determined by FACS. SG500-dNKmu induced the greatest selective apoptosis in cancer cells. 
$\mathbf{A}$
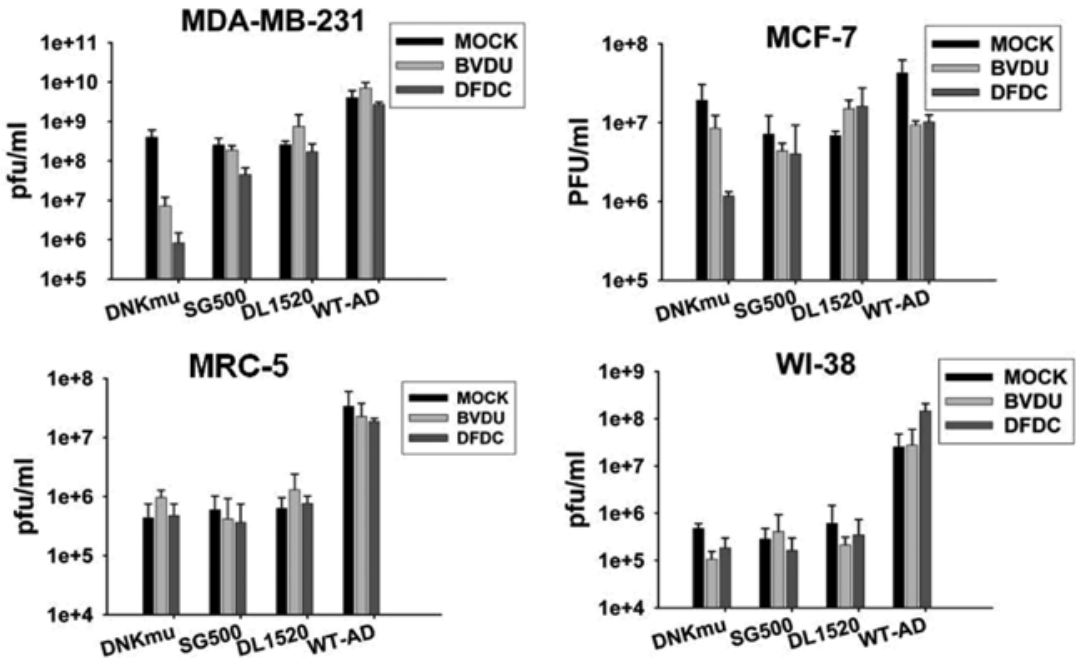

B

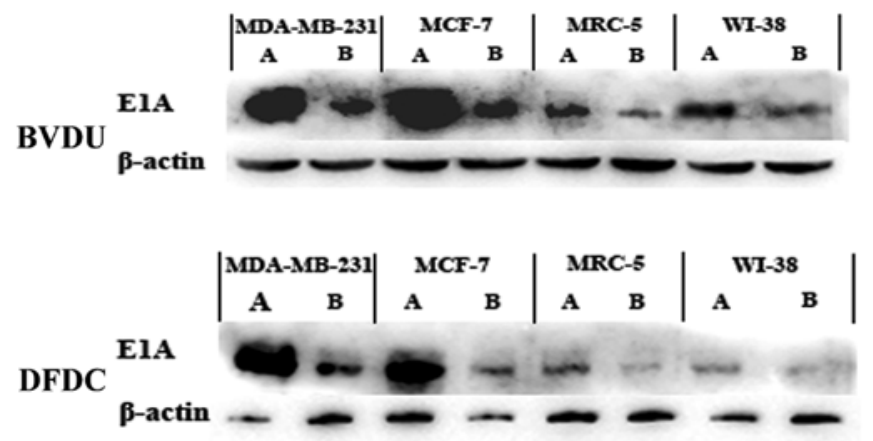

Figure 5. SG500-dNKmu enhanced the inhibition effect of BVDU or DFDC on viral replication in cancer cell lines MDA-MB-231, MCF-7 and in normal cell WI-38 and MRC-5. (A) Replication in cells was analyzed after 5 days of treatment with viruses alone (MOI 10) and in combination with $1 \mu$ M of DFDC. Drugs were added after virus infection (2 days). Both cells and media were analyzed by TCID50 assays. (B) Western blot assay of E1A gene expression by SG500dNKmu, SG500, Mock with or without BVDU, DFDC. SG500-infected cells and mock were used as a control.

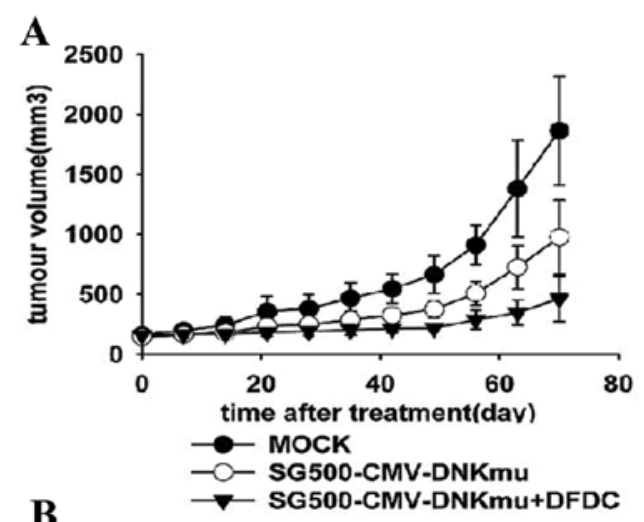

B

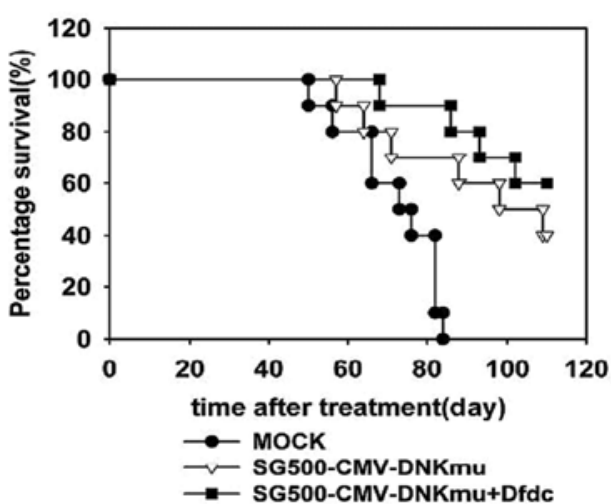

with DFDC were at the lowest levels in both MDA-MB-231 and MRC-5 cells (Fig. 5B), not obvious in that with BVDU. SG500-dNKmu followed by the addition of DFDC attenuated E1A expression of adenovirus obviously in cancer cell lines, but no clear lane of E1A was detected in MRC-5 cells, except for wild-type Ad. These results show that viral E1A expression is not sufficient to overcome the DFDC-induced arrest in response to DNA damage causing attenuation of replication. As expected, viral replication was decreased in the presence of this potent DNA-polymerase inhibitor, even at low concentrations.

Antitumoral efficacy of Dm-dNKmu with DFDC in MDA-MB231 xenograft in vivo. The potency of the combination

Figure 6. In vivo antitumor potential of SG500-dNKmu and SG500 in breast cancer MDA-MB-231 xenograft mouse model. Xenografted mice $(8$ in each group) were given intratumoral injections of the indicated viruses on 5 alternate days with a total dose of $10^{9} \mathrm{pfu} /$ mouse. (A) Tumor volume $\left(\mathrm{mm}^{3}\right)$ was monitored up until day 60 (the day they had to be sacrificed) and up until day 29 for the PBS-treated control (mock) group. $\mathrm{p}<0.01$ for combination treatments compared with either single-agent treatment and $\mathrm{p}<0.05$ for each treatment compared with mock-treated animals. Significance was determined by one-way ANOVA analysis. (B) Kaplan-Meier curves for the indicated treatment groups. All combinations were significantly different from single-agent treatments with $\mathrm{p}<0.05$. 
treatments was evaluated in an in vivo MDA-MB-231 xenograft model with subcutaneous tumors treated with suboptimal doses of SG500-dNKmu, SG500 and other control viruses with or without DFDC (Fig. 6A and B). In animals treated with a virus dose at $1 \times 10^{9} \mathrm{pfu}$, median survival was 40 days for SG500-dNKmu. However, when SG500 and SG500-dNKmu in combination with $5 \mathrm{mg} / \mathrm{kg}$ of DFDC was studied, time to progression was prolonged to $>60$ days. Statistically significant antitumoral efficacy in terms of tumor size was observed in SG500-dNKmu and SG500 with DFDC-treated groups $\left(1,032.2 \pm 613.1,844.6 \pm 165.3 \mathrm{~mm}^{3}\right)$ compared with the virus $\left(2,033.3 \pm 410.2\right.$ and $\left.2,754.6 \pm 332.9 \mathrm{~mm}^{3}\right)$ and mock control group $\left(2,766.76 \pm 431.54 \mathrm{~mm}^{3}\right)$, respectively, $\mathrm{p}<0.05$. The SG500-dNKmu-treated group appeared to be the most efficient in retarding tumor growth with the longer survival period.

\section{Discussion}

We found that the replication capacity of SG500-dNKmu could specifically replicate and cause significant CPE in breast cancer cells, while showing a very low effect in normal cells. In addition, SG500-dNKmu showed markedly strong enzyme activity in breast cancer cells and significantly weak in normal cells, suggesting that this oncolytic adenovirus can target malignancy with high specificity. In the MTT assay, the nucleoside analogs show low toxicity to untransduced cells, but the cells expressing Dm-dNKmu are highly sensitive to the compound, especially for DFDC, and the FACSCalibur confirmed that the cytotoxic effect of SG500-dNKmu combined with DFDC was mainly due to apoptotic events.

SG500-dNKmu was supposed to express enzymatical activity of $\mathrm{dNKmu}$ increased with the adenovirus genome amplification. Interestingly, there was no obviously potent increase in cell death in response to the combined treatment depended on virus, even attenuation of replication with SG500$\mathrm{dNKmu}$ in gastric cancer cells combined with $1 \mu \mathrm{M}$ of DFDC was observed 5 days post-infection. We considered that the DFDC conversion to its triphosphates form (TP) phosphorylated by dNKmu may lead to more apoptosis in the cancer cells and the adenovirus, as shown in our western blot analysis of adenovirus E1A protein and titer assays. The replication of adenovirus could be controlled, to some extent, by certain types of dNKmu that possess nucleoside analogs to protect the normal cells. DFDC itself also could block replication of viruses due to DFDC-induced delay in G1/S-cell cycle progression, with repression of cyclin $\mathrm{E}$ and cdc25A (13-15). In a more recent publication by Morris and Wildner, using wild-type replicating adenoviruses containing an HSV-TK transgene, no augmentation of killing was observed after the addition of GCV (16). The controversial conclusion could be explained by the GCV-TP inhibiting the virus vector expression of HSV-TK continuously through inducing the death of virus, as is our finding.

Although SG500 combined with DFDC showed no less killing activity than SG500-dNKmu in the breast carcinoma cell line MDA-MB-231 or the tumor xenograft mouse model, the activated DFDC played an important role in inhibiting viral replication. Activated DFDC actually is limited by low selectivity toward the target cells and low transduction efficiency. Futhermore, severe side-effects resulting from excessive replication were the key obstacles of CRCAds in the clinical treatment of cancer gene therapy (17-19). Expression of adenovirus early transcription gene E1A could be controlled, to some extent, and was associated with apoptosis in the majority of SG500$\mathrm{dNKmu/DFDC-treated} \mathrm{tumor} \mathrm{cells,} \mathrm{even} \mathrm{when} \mathrm{the} \mathrm{adenovirus}$ vector SG500 amplification in the normal cells expressed suicide gene to produce unwanted effects.

In conclusion, we have developed a replicative, Dm-dNKmu-armed adenovirus, SG500-dNKmu, which demonstrated that cancer-specific killing activity could be enhanced by pro-drugs administration. The combination therapy synergistically induced growth apoptosis in human breast cancer cells while suppressing the replication of the adenovirus in vitro. DFDC with less toxic doses under dNKmu catalysis enhanced cytotoxicity and had a synergistic effect of suppressing excessive adenovirus replication. Combining the $\mathrm{dNKmu}$ therapy with other antimetabolites to achieve synergistic effects may be another approach to enhance the efficiency of nucleoside kinase suicide gene therapy. Even though mechanisms of synergetic effects still need to be studied and preventive strategies are being entertained, the ultimate clinical use of gene therapy for improving breast cancer treatment would most likely be in combination with surgery, radiation, or chemotherapy.

\section{Acknowledgements}

This study was supported by grants from the Hi-Tech Research Development Program of China (863 Program, 2006AA02Z493) and the National Natural Science Foundation of China (no. 81071900 and 81172199). We appreciate the support of Professor Qijun Qian (Laboratory of Gene and Viral Therapy, Eastern Hepatobiliary Surgical Hospital, Second Military Medical University, China) in constructing the adenovirus vectors.

\section{References}

1. Zheng X, Johansson M and Karlsson A: Retroviral transduction of cancer cell lines with the gene encoding Drosophila melanogaster multisubstrate deoxyribonucleoside kinase. J Biol Chem 275: 39125-39129, 2000 .

2. Zheng X, Johansson M and Karlsson A: Bystander effects of cancer cell lines transduced with the multisubstrate deoxyribonucleoside kinase of Drosophila melanogaster and synergistic enhancement by hydroxyurea. Mol Pharmacol 60: 262-266, 2001.

3. Zheng $X$, Johansson $M$ and Karlsson A: Nucleoside analog cytotoxicity and bystander cell killing of cancer cells expressing Drosophila melanogaster deoxyribonucleoside kinase in the nucleus or cytosol. Biochem Biophys Res Commun 289: 229-233, 2001

4. Betham B, Shalhout S, Marquez VE and Bhagwat AS: Use of Drosophila deoxynucleoside kinase to study mechanism of toxicity and mutagenicity of deoxycytidine analogs in Escherichia coli. DNA Repair (Amst) 9: 153-160, 2010.

5. Munch-Petersen B, Knecht W, Lenz C, Sondergaard L and Piskur J: Functional expression of a multisubstrate deoxyribonucleoside kinase from Drosophila melanogaster and its C-terminal deletion mutants. J Biol Chem 275: 6673-6679, 2000.

6. Sokkar P, Sathis V and Ramachandran M: Computational modeling on the recognition of the HRE motif by HIF-1: molecular docking and molecular dynamics studies. J Mol Model 18: 1691-700, 2012.

7. Lee KJ, Lee KY, Lee YM: Downregulation of a tumor suppressor RECK by hypoxia through recruitment of HDAC1 and HIF-1alpha to reverse HRE site in the promoter. Biochim Biophys Acta 1803: 608-616, 2010.

8. Mikkelsen NE, Munch-Petersen B and Eklund H: Structural studies of nucleoside analog and feedback inhibitor binding to Drosophila melanogaster multisubstrate deoxyribonucleoside kinase. FEBS J 275: 2151-2160, 2008. 
9. Yu de B, Zhong SY, Yang M, Wang YG, Qian QJ, Zheng S and Liu XY: Potent antitumor activity of double-regulated oncolytic adenovirus-mediated ST13 for colorectal cancer. Cancer Sci 100: 678-683, 2009

10. Beltinger C, Fulda S, Kammertoens T, Meyer E, Uckert W and Debatin KM: Herpes simplex virus thymidine kinase/ganciclovir-induced apoptosis involves ligand-independent death receptor aggregation and activation of caspases. Proc Natl Acad Sci USA 96: 8699-8704, 1999.

11. Wildner O and Morris JC: The role of the E1B $55 \mathrm{kDa}$ gene product in oncolytic adenoviral vectors expressing herpes simplex virus-tk: assessment of antitumor efficacy and toxicity. Cancer Res 60: 4167-4174, 2000.

12. Sandrini MP, Clausen AR, On SL, Aarestrup FM, MunchPetersen B and Piskur J: Nucleoside analogues are activated by bacterial deoxyribonucleoside kinases in a species-specific manner. J Antimicrob Chemother 60: 510-520, 2007.

13. Jordheim LP, Galmarini CM and Dumontet C: Gemcitabine resistance due to deoxycytidine kinase deficiency can be reverted by fruitfly deoxynucleoside kinase, DmdNK, in human uterine sarcoma cells. Cancer Chemother Pharmacol 58: 547-554, 2006.
14. Zhang ZL, Zou WG, Luo CX, et al: An armed oncolytic adenovirus system, SG500-gene, demonstrating potent antitumoral efficacy. Cell Res 13: 481-489, 2003.

15. Cappella P, Tomasoni D, Faretta M, et al: Cell cycle effects of gemcitabine. Int J Cancer 93: 401-408, 2001.

16. Morris JC and Wildner O: Therapy of head and neck squamous cell carcinoma with an oncolytic adenovirus expressing HSV-tk. Mol Ther 1: 56-62, 2000

17. Heilbronn R and Weger S: Viral vectors for gene transfer: current status of gene therapeutics. Handb Exp Pharmacol 197: 143-170, 2010.

18. Nayak S and Herzog RW: Progress and prospects: immune responses to viral vectors. Gene Ther 17: 295-304, 2010.

19. Büning H, Perabo L, Coutelle O, Quadt-Humme S and Hallek M: Recent developments in adeno-associated virus vector technology. J Gene Med 10: 717-733, 2008. 